\title{
Acta Paediatrica Japonica
}

Official Journal of Japan Pediatric Society

Vol. 33 No. 3 June 1991

(Acta Paediatr Jpn 1991; 33: $279 \sim 283$ )

\section{Guest Lectures}

\section{Recent Advances in Childhood Diarrhoeal Diseases}

\author{
Michael Gracey, M.D., Ph.D.
}

Aboriginal Health Unit, Health Department of Western Australia, Perth Australia

Over the past 20 years there have been many important advances in knowledge about diarrhoeal diseases of children. Of these, the development of oral rehydration therapy (ORT), has been outstanding. This is based on the observation that intestinal sodium transport is enhanced by glucose transport in the small intestine and that this sodium-coupled mechanism for glucose and water transport remains intact in the enterotoxigenic diarrhoeas despite the net secretory effects of bacterial enterotoxins on the small intestinal epithelium. The World Health Organisation has adopted ORT and strongly promoted its application for treatment of diarrhoeal diseases, particularly in developing countries. This has resulted in a drop in diarrhoeal disease mortality and the application of this treatment has been described in an Editorial in the Lancet as "potentially the most important medical advance this century"[1].

\section{Introduction}

Another major advance has been the discovery of "newer" micro-organisms, including viruses and enterotoxigenic bacteria, which cause diarrhoea in children. Before the 1970's most such episodes were undiagnosed. The com-

Received July 7, 1990

Correspondence address: Michael Gracey, M.D., Ph.D., Aboriginal Health Unit, Health Department of Western Australia, 189 Royal Street, East Perth, WA, 6004 Australia monest bacterial and viral enteric pathogens found in a recent 12-month, hospital-based study of almost one thousand children with acute diarrhoea in Perth are shown in Fig. 1. The so-called "newer" infective agents include viruses, enterotoxigenic bacteria, Aeromonas, Campylobacter, Yersinia and some parasites, notably Cryptosporidium.

\section{BACTERIA}

Enteropathogenic E.coli (EPEC)

EPEC are important in infants and children 
in developing countries. particularly where standards of living and hygiene are low. Detection of EPEC by serotyping became widely used in the 1960's but lost favour in the late 1970 's when it was found that many strains of EPEC do not produce toxins. However. some EPEC strains which are non-invasive and which do not produce heat-labile toxin (LT) or heat-stable toxin (ST) can cause diarrhoea, perhaps through production of other toxins.

\section{Enterotoxigenic E.coli (ETEC)}

Strains of E.coli which produce enterotoxins (i.e. enterotoxigenic) are very important causes of diarrhoea, particularly in infants and young children. Poor standards of hygiene are important and cross-infection and inadequate nursing practices can encourage the spread of ETEC in hospitals; overcrowded nurseries for neonates and older infants are important potential sources. Contaminated food and water can be responsible for outbreaks in older patients.

\section{Enteroinvasive E.coli (EIEC)}

Some strains of E.coli resemble Shigella spp. and cause similar dysenteric clinical features with diarrhoea accompanied by blood and mucus. Invasiveness of the organism can be demonstrated by the keratoconjunctivitis guinea pig model (the Sereny test) or by showing invasion of cultured cells in vitro.

\section{Enteroadherent E.coli (EAEC)}

Mathewson et al (1985) found that certain strains of E.coli did not produce recognised enterotoxins, did not belong to recognised EPEC serogroups but adhered to HEp-2 cells cultured in vitro and were associated with diarrhoea in travellers to Mexico. Overall, they were found in almost $15 \%$ of patients with diarrhoea (compared to $7.6 \%$ without diarrhoea) and occurred in more that $30 \%$ of diarrhoeal patients in whom no other known enteric pathogens were identified. These EAEC are significant causes of diarrhoea in travellers; their role in other types of acute diarrhoea is not yet known.

\section{Campylobacter}

This organism has become recognised in the past 10 years as a major cause of acute diarrhoea in children [11]. The typical clinical picture in children includes prodromal fever, muscle pain, headache and abdominal pain which after a few days leads to colicky pain with diarrhoea and then bloody diarrhoea with an inflammatory exudate occurring a day or two later. Patients can be infected even in the first month of life.

The rate of isolation of Campylobacter from human faeces is closely related to standards of hygiene. In industrialised countries the isolation rate is very low-less than $1 \%-$ while in developing countries it is much higher. Campylobacteriosis, like salmonellosis, is a zoonotic infection and various animals including dogs, cats, poultry, cattle and sheep can be infected; several routes of transmission are possible including person-to-person, animal-toperson, or through contaminated food, milk or water.

\section{Aeromonas}

Reports of Aeromonas-associated diarrhoea have been made from many parts of the world including North America, South America, Europe, Australia, India, Africa and South East Asia and it is now becoming increasingly recognised as an enteric pathogen for humans [5].

Isolation rates for faecal Aeromonas vary widely from place to place, probably because of differences in methods used for their isolation, differences in ways of selecting patients and true geographical variations in their incidence.

Aeromonas species are widespread in the environment and occur in animals including frogs, fish, reptiles and laboratory animals. Contaminated waters are likely to be sources for human gastrointestinal infections whether they are recreational waters, such as swimming pools, lakes or estuaries, or whether they are domestic water supplies. Aeromonas gastroenteritis is commonest in the warmest months of the year [5].

Acromonas gastroenteritis has two age 
peaks; in young children and in adults 60 years of age and over. In children there are three main clinical pictures; watery diarrhoea for several days; chronic, watery dysentery or inflammatory bowel disease [9].

\section{Plesiomonas shigelloides}

Plesiomonas shigelloides is known to cause epidemic outbreaks of watery diarrhoea and seems to be important in India, other tropical areas, and Japan. The disease is thought to be water-borne and although some strains are toxigenic, the pathogenesis of the infection is obscure.

\section{Vibrio parahaemolyticus}

Infections with $V$. parahaemolyticus seem to affect adults more than children and tend to occur in summer, perhaps because of infection through contaminated food, seawater or consumption of contaminated seafood [3]. The disease is usually characterised by watery diarrhoea although dysenteric symptoms, with blood and mucus in the loose stools, may occur. Important outbreaks have occurred in Japan, India and South East Asia [3] and have also been reported from the United States.

\section{Clostridium difficile}

Antibiotic-induced enterocolitis can affect infants and children and characteristically causes fever, vomiting, abdominal pain and sometimes bloody diarrhoea or leucocytosis. The diagnosis can be confirmed by sigmoidoscopy and the demonstration of cytotoxic $C$. difficile in the stools. However, toxigenic $C$. difficile often occurs in the faeces of normal infants under 5 months of age which makes the diagnosis difficult in infants.

\section{Yersinia}

Yersinia enteritis is usually due to $Y$. enterocolitica and most instances occur in children less than seven years of age. The illness is usually associated with fever, vomiting, abdominal pain and diarrhoea, often with blood, mucus and an inflammatory exudate. The clinical features can suggest inflammatory bowel disease and older patients may have extra-intestinal symptoms such as iritis, arthritis, conjunctivitis and erythema nodosum.

\section{VIRUSES}

\section{Rotavirus}

This is the best known and probably the commonest virus which infects humans and is responsible for much vomiting and diarrhoeal disease in infants and young children.

Rotavirus gastroenteritis is predominantly a disease of children under 2 years old with a peak incidence about 9-12 months [4] and with most episodes occurring during winter in temperate climates. In the tropics rotavirus enteritis tends to occur throughout the year although seasonal increases have been reported in both the dry season and the wet. Outbreaks in neonates can also occur throughout the year.

Although rotavirus infection has been widely accepted as a major cause of gastroenteritis in infants and young children, asymptomatic excretion is not uncommon [16] and the $80-90 \%$ prevalence of antibody in most areas where surveys have been carried out suggests that many mild or undiagnosed infections occur which do not cause overt disease [13]. This applies to our experience in Western Australia where rotavirus was not detected significantly more often in children with acute diarrhoea (12.7\%) than it was in controls (11.1\%) [5] (Fig. 1).

Rotavirus infection is transmitted by the faecal-oral route and spread can occur within families; adults may acquire the infection without symptoms. Nosocomial infections can occur and may be related to the massive shedding of viruses which occurs during the several days of active infection and to the ability of the virus to survive on environmental surfaces for weeks.

The incubation period of rotavirus infection is 2 to 3 days; this is followed by fever, then diarrhoea and vomiting which is sometimes preceded or accompanied by upper respiratory tract symptoms. The stools are usually fluid, often quite large and are usually without blood or mucus. Diarrhoea is usually worst on the second or third day and the illness is usually 
self-limiting within a week or so. Because of mucosal involvement, secondary lactose intolerance is common but usually resolves within 2 to 4 weeks, [7].

\section{Norwalk-like agents}

An agent which caused an outbreak of diarrhoea and vomiting in Norwalk, Ohio, in 1968 became known as the Norwalk agent. Similar agents found elsewhere have been called Hawaii virus, Montgomery County virus, Marin County virus, Ditchling virus and Parramatta virus (23). Norwalk virus is thought not to be an important cause of severe gastroenteritis but it can affect older subjects of any age. It may be relatively more important in infants and young children in developing countries [10].

\section{Adenovirus}

Morphologically typical adenoviruses have been isolated by many investigators from faecal specimens of children with acute diarrhoea. These so-called fastidious adenoviruses appear to be emerging as the next most common viral agents, after rotavirus, in childhood gastroenteritis [4].

\section{Coronaviruses}

Faecal coronaviruses have been reported from patients with diarrhoea, but their significance is unclear.

\section{Astroviruses}

These are small round viruses, 28 to $30 \mathrm{~nm}$ in diameter with a characteristic star-like appearance to which they owe their name. Their isolation has been identified with diarrhoea in $80 \%$ of children excreting the virus [12].

\section{Calicivirus}

These 31 to $40 \mathrm{~nm}$ particles with cup-shaped depressions on their surface have been linked with outbreaks of childhood enteritis, so-called "winter vomiting" [6], but are uncommon with children hospitalised because of gastroenteritis.

\section{PARASITES}

Giardia lamblia is widespread and an important cause of gastrointestinal symptoms, including diarrhoea and abdominal discomfort in children, particularly where standards of community and personal hygiene are unsatisfactory [15].

Diarrhoea due to Cryptosporidium infections has recently become recognised as an important problem in apparently healthy children and has emerged as a significant cause of illness in children attending day care centres, particularly in Europe and the United States. Such centres are increasingly being recognised as reservoirs of infectious agents such as respiratory tract viruses, hepatitis A, Staphylococcus, Streptococcus, Haemophilus influenzae type B and Neiserria meningitidis as well as enteric pathogens. The incidence of diarrhoea in children under 3 years in day care centres was reported as 42 episodes per child-months or 3.5 illnesses per child per year [2]. There is a significant secondary attack rate (almost 40\%) among household contacts exposed to children from the day care centres; these findings illustrate the potential for common-source outbreaks in groups of children who are not fully toilet-trained and spend significant periods of time together and how this can provide a reservoir for transmission of the parasite into the community. This has been commented on recently elsewhere [8].

\section{References}

1. Anonymous. Water with sugat and salt (Editorial) Lancet 1978; ii: 300-301.

2. Bartlett AV, Moore M, Garry GW et al. Diarrheal illness among infants and toddlers in day care centers. I1. Comparison with day care houses and households. J Pediatr 1985; 107: 503-509.

3. Blake PA, Weaver RE, Hollies DG. Diseases of humans (other than cholera) caused by vibrios. Ann Rev Microbiol 1980; 34: 34I-367.

4. Brandt CD, Kim HW, Rodriguez WJ et al. Pediatric viral gastroenteritis during eight years of study. J Clin Microbiol 1983; 18: 71-78.

5. Burke V, Gracey $M$, Robinson $J$ et al. The microbiology of childhood gastroenteritis: Aeromonas species and other infective agents. J Infect Dis 1983; 148: 68-74.

6. Cubitt WD, McSwiggan DA, Moore W. Winter vomiting disease caused by calicivirus. J Clin Pathol 1979; 32: 786-793. 
7. Davidson GP, Goodwin D, Robb TA. Incidence and duration of lactose malabsorption in children hospitalised with acute enteritis: study in a wellnourished urban population. J Pediatr 1984; 105 : $587-590$.

8. Gracey M. Gastrointestinal infection in children. Curr Op Gastroenterol 1990; 6: 118-123.

9. Gracey M, Burke V, Robinson J. Aeromonas associated gastroenteritis. Lancet 1982; ii: 1304-1306.

10. Kapikian AZ, Greenberg HB, Wyatt RG et al. The Norwalk group of viruses-agents associated with epidemic viral gastroenteritis. In: Virus Infections of the Gastrointestinal Tract. New York, 1982 Marcel Dekker, P.147-177.

11. Karmali MA, Fleming PC. Campylobacter enteritis in children. J Pediatr 1979; 94: 527-532.

12. Madelay CR. Viruses and diarrhoea? problems in proving causation. In: De la Manza L, Person EM, eds. Medical Virology II. New York, 1983, Elsevier Science Publishing Co., P.81-109.

13. Madeley CR. Viruses and diarrhoea: some current preoccupations. Curr Op Gastroenterol 1986; 2: 1149-1150.

14. Mathewson IJ, Johnson PC, DuPont HL et al. A newly recognised cause of travelers' diarrhea: enteroadherent Escherichia coli. J Infect Dis 1985; 151: 471-475.

15. Meloni BP, Lymbery AJ, Gracey $M$ et al. High prevalence of Giardia in children from a Western Australian Aboriginal community. Med J Aust 1988; 149: 715.

16. Murphy AM, Albrey MB, Crewe EB. Rotavirus infections of neonates. Lancet 1977; ii: 1149-1150. 